\title{
Implementation of slow magnetic relaxation in a SIM-MOF through a structural rearrangement
}

Received 00th January 20xx, Accepted 00th January 20xx

DOI: $10.1039 / x 0 x x 00000 x$

www.rsc.org/

\author{
Javier Castells-Gil, ${ }^{a}$ José J. Baldoví ${ }^{b}$, Carlos Martí-Gastaldo a and Guillermo Mínguez Espallargas*a
}

Here we report the structural flexibility of a Dy-based Single-lon Magnet MOF in which its magnetic properties can be modified through a ligand substitution process involving an increase of the charge density of the coordination environment.

\section{Introduction}

Metal-Organic Frameworks (MOFs) are exciting crystalline molecule-based materials that, since their emergence around 30 years ago, have attracted a great interest due to their ample range of possible applications. ${ }^{1,2}$ Their high chemical versatility and structural tunability also offer additional features like the rational design of multifunctional architectures by combination of two or more functionalities within the same framework. ${ }^{3}$ In this regard, magnetic MOFs can be of interest in sensing, magnetic sequestration and stimuli-responsive materials. ${ }^{4-6}$ Long-range magnetic ordering is restricted to bridging linkers that mediate strong enough superexchange interactions, often not accessible with conventional MOF-forming polyaromatic linkers. Single-molecule magnets (SMM) are arguably better fitted to this purpose, as the magnetic response is intrinsic to discrete mononuclear or polynuclear metal complexes. These systems display a number of characteristic phenomena, such as magnetic hysteresis at low temperature and macroscopic quantum tunnelling of magnetization ${ }^{7}$ and they have been considered as attractive candidates in the fields of molecular spintronics and quantum computing. ${ }^{8}$ The incorporation of SMMs as magnetic nodes of MOFs has proven an efficient way for the three dimensional organization of these interesting entities, 9,10 and even modulating their magnetic response through the accommodation of different guests within the pores. ${ }^{11}$

One of the major difficulties in the design of SMM-MOFs relies on the retention of the slow magnetic relaxation of the polynuclear clusters upon formation of an extended systems. We have recently shown that replacing the SMM clusters by mononuclear lanthanoid analogues, also known as single-ion magnets (SIMs), can enormously simplify this issue ${ }^{12}$ with the formation of SIM-MOFs. In fact, SIMs have been one of the hottest topics in molecular magnetism. ${ }^{13-15}$ Although a precise

\footnotetext{
a. Instituto de Ciencia Molecular (ICMol), Univ. de Valencia, C/Catedrático Beltrán 2, E-46980 Paterna, Spain.E-mail: guillermo.minguez@uv.es.

b. Max Planck Institute for the Structure and Dynamics of Matter, Luruper Chaussee 149, D-22761 Hamburg, Germany.

† Footnotes relating to the title and/or authors should appear here.

Electronic Supplementary Information (ESI) available: [details of any supplementary information available should be included here]. See DOI: 10.1039/x0xx00000x
}

description of the spin dynamics of these molecular entities is still very challenging due to the major role played by vibrations, ${ }^{16}$ a large number of theoretical efforts have permitted to guide the synthesis of new SIMs, $15,17,18$ achieving record values of $T_{B}=60 \mathrm{~K} .{ }^{19,20}$ However, the number of Ln-MOFs exhibiting SMM behavior still remains limited, ${ }^{21}$ with only a few examples reported so far. ${ }^{12,22-26}$

Herein, we investigate the structural flexibility of a novel Dybased MOF, namely MUV-4 (MUV = Materials of University of Valencia), in which SMM behaviour can be implemented through a controlled desolvation process that modifies the coordination environment of the Dy(III) ions. MUV-4 is built from 3,3',5,5'-azobenzene-tetracarboxylic acid ( $\mathrm{H}_{4} \mathrm{abtc}$ ) and dimeric Dy(III) units, and is capable of undergoing a ligand substitution upon desolvation, resulting in the appearance of slow magnetic relaxation by changing the charge density around the Dy(III) ions.

\section{Results and Discussion}

\section{Synthesis and Characterization}

$\mathrm{H}_{4}$ abtc was synthesized according to a reported procedure (Supporting Information Section 2). ${ }^{27}$ Reaction of Dy $\left(\mathrm{CH}_{3} \mathrm{COO}\right)_{3} \cdot 6 \mathrm{H}_{2} \mathrm{O}$ with $\mathrm{H}_{4}$ abtc in a mixture of $\mathrm{H}_{2} \mathrm{O}$ and acetic acid at $160^{\circ} \mathrm{C}$ yields single crystals of MUV-4a of around $100 \mu \mathrm{m}$ size (Figure S1) of formula [Dy $\left.\left(\mathrm{H}_{2} \mathrm{O}\right)_{2}(\mathrm{Habtc})\right] \cdot 1.7 \mathrm{H}_{2} \mathrm{O}$. Singlecrystal X-ray diffraction (SCXRD) analysis shows that this solid crystallizes in the triclinic space group $P$-1. The structure of MUV-4a is built upon dimeric Dy $y_{2}$ units of formula $\mathrm{Dy}_{2}\left(\mathrm{H}_{2} \mathrm{O}\right)_{4}(\mu-$ $\left.\mathrm{O}_{2} \mathrm{C}\right)_{2}\left(\mathrm{O}_{2} \mathrm{C}\right)_{4}\left(\mathrm{HO}_{2} \mathrm{C}\right)_{2}$ (Figure 1), with two coordinated water molecules per Dy ion, connected by partially deprotonated $\mathrm{Habtc}^{3-}$ anions. Each Dy(III) ion in the secondary building unit (SBU) is octacoordinated featuring a square antiprism geometry. Both Dy atoms are kept together by two bridging carboxylate groups with a Dy-Dy distance of $5.462 \AA$. Selfassembly of this SBU with Habtc ${ }^{3-}$ linkers yields a laminar structure whose layers extend along the $b c$ plane. Hydrogen bonding of the coordinated water molecules of adjacent layers keep the layers together forming a 3D structure with 1D micropores extending along the $c$ axis (Figure 1, S2b,c). These channels are also filled with water molecules that participate actively in the interlayer $\mathrm{H}$-bonding (Figure S2c).

Phase purity of the material was confirmed by means of powder $X$-ray diffraction (PXRD) and thermogravimetric (TG) analysis (Figure S5,11). TG analysis of MUV-4a suggests that the first weight loss at around $100{ }^{\circ} \mathrm{C}$ corresponds to the loss of all the water molecules in the structure, i.e. the coordinated water 
molecules and those present in the pores, as shown by the good correlation between the experimental and the calculated weight loss (exp: $11.22 \%$; calc: $11.39 \%$ ).

\section{Structural rearrangement}

We hypothesized that the loss of the coordinated water molecules would create coordinatively vacant positions in the Dy(III) ions that would trigger a structural reorganization in order to re-occupy the Dy(III) open metal sites. Indeed, a structural transition was observed when heating the material above $100^{\circ} \mathrm{C}$. It was found that MUV-4a undergoes a structural transformation with retention of the long-range order. ${ }^{28}$ Thus, we have been able to successfully characterize by SCXRD the dehydrated form, namely MUV-4b of formula [Dy(Habtc)] $2 \mathrm{H}_{2} \mathrm{O}$. MUV-4b also crystallizes in the triclinic space group $P-1$, although it no longer displays the layered structure observed in MUV-4a; conversely, it features instead threedimensional connectivity. This change is linked to the connection of Dy 2 SBUs from adjacent layers to form infinite Dycarboxylate chains linked together by $\mathrm{Habtc}^{3-}$ anions, generating microporous 1D channels along the $a$-axis (Figure 2, S3) which are filled with water molecules from the ambient. Dy(III) ions in MUV-4b retain the 8-coordinated environment, although with a triangular dodecahedron geometry (Figure S20), slightly different than that observed in MUV-4a. However, all oxygen atoms belong now to carboxylate groups (i.e. there is no coordinated water molecules).

A probable mechanism through which the structural transformation is taking place is depicted in Figure 2. We hypothesized that the vacant positions created in the Dy(III) ions upon removal of the coordinating water molecules of MUV-4a, are filled by the free $\mathrm{O}$ atom of the deprotonated carboxylate at the same time that this $\mathrm{O}$ atom links to the other vacant position of the nearest Dy atom of an adjacent layer (red and blue arrows in Figure 2), thus creating a bridge between Dy atoms of different layers. For this transition to happen, the Dy 2 SBUs of MUV-4a must rotate at a certain angle $\left(c a .27^{\circ}\right)$. This would induce a shift of the planes defined by the organic ligands to stack one upon the other.

Importantly, we could identify a second crystalline phase arising from the desolvation of MUV-4a, namely MUV-4c, which was also successfully characterized by SCXRD. MUV-4c presents a microporous 3D structure similar to that encountered in MUV$\mathbf{4 b}$ but with major changes to the coordination sphere of Dy atoms, which are 7-coordinated in this case (Figure S4). Thus, this suggests that the structural transformation observed in MUV-4a to form MUV-4b occurs via previous transformation in MUV-4c. In other words, MUV-4c seems to be an intermediate of the reaction. Note that the Dy-Dy distances for the nearest Dy neighbours of different layers vary from $6.294 \AA$ in MUV-4a, to $4.051 \AA$ in MUV-4b for the same pair of Dy atoms, whereas the Dy-Dy distances in MUV-4c are all $4.737 \AA$, i.e. an intermediate value between those found in MUV-4a and MUV4b (Table S2).

We also tested the water stability of the new crystalline phases. MUV-4a showed an excellent water stability in water in a wide range of $\mathrm{pH}$ values or boiling water without any sign of degradation as confirmed by PXRD measurements (Figure 3a). As expected for the three-dimensional networks MUV-4b and MUV-4c, there was no sign of hydrolytic degradation of these crystalline phases between $\mathrm{pH} 2$ or 11 nor after soaking in boiling water overnight, remaining highly crystalline. However, we observed a shift of the main peak from $7.01^{\circ}$ (for MUV-4b) and $7.81^{\circ}$ (for MUV-4c) towards $6.25^{\circ}$ in all cases, thus forming a new crystalline phase, namely MUV-4d (Figure $3 \mathrm{~b}$ ). This crystalline phase returns exclusively to MUV-4b (Figure 3c) only when heated above $100{ }^{\circ} \mathrm{C}$. It should be noted that a small unidentified impurity is observed in the PXRD of MUV-4b at around $12^{\circ}$ (Figure S7). Nevertheless, the transition between MUV-4b and MUV-4d happens in a reversible manner without estimable loss of crystallinity after several cycles (Figure S8), characteristic of MOFs with adaptable structures. ${ }^{29}$ However, despite many efforts, the structure of MUV-4d could not be determined.

\section{Magnetic Properties}

The similar coordination environment of the Dy(III) ions in MUV4a and MUV-4b might suggest the presence of comparable magnetization dynamics at low temperature. Static magnetic measurements $(d c)$ of MUV-4a and MUV-4b were performed between 2 and $300 \mathrm{~K}$ under an applied field of $1000 \mathrm{G}$ (Figure 3, S16). The $\chi_{m} T$ values at room temperature are 14.08 and 14.15 emu.K. $\mathrm{mol}^{-1}$ for MUV-4a and MUV-4b, respectively, which are very close to the expected value for the ${ }^{6} \mathrm{H}_{15 / 2}$ multiplet of $\mathrm{Dy}^{3+}$ (14.17 emu $\cdot \mathrm{K} \cdot \mathrm{mol}^{-1}$ ). The observed drop of the $\chi_{m} T$ upon cooling is primarily ascribed to the depopulation of the highest crystal field levels, as expected for anisotropic lanthanide ions. The presence of weak dipolar interactions between neighbouring lanthanide centres has been discarded after examining the $\mathrm{Gd}$ analogue of MUV-4a, which shows a constant $\chi_{m} T$ value in the same range of temperatures (Figure SI15).
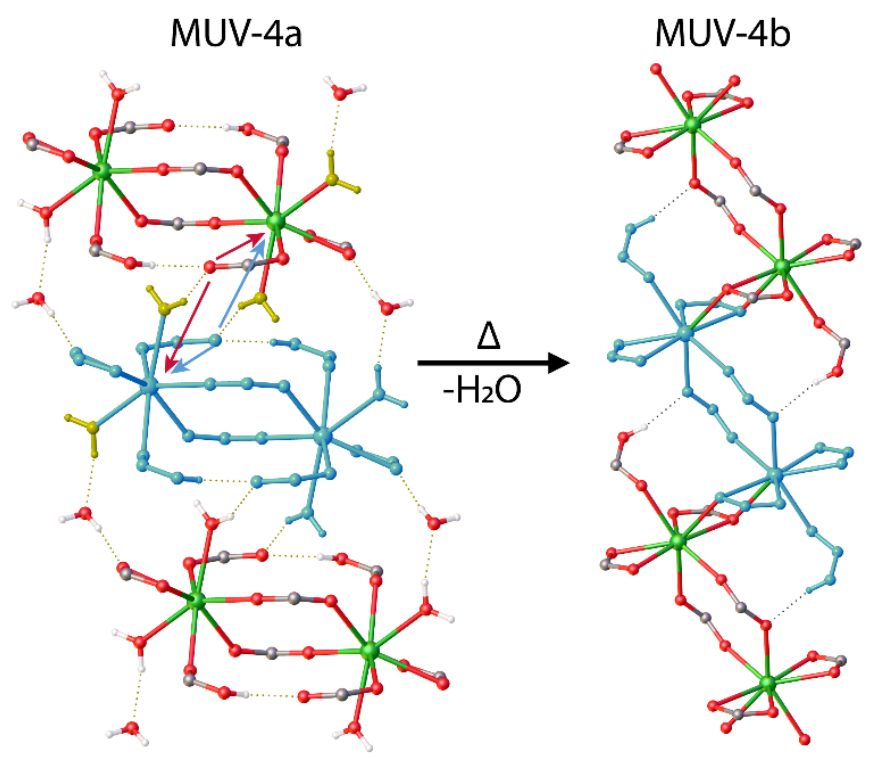

Figure 1 - Proposed mechanism for the transformation of MUV-4a to MUV-4b. The arrows indicate the movement of the carboxylate groups that occupy the vacant positions left by the extrusion of water molecules (shown in yellow). 

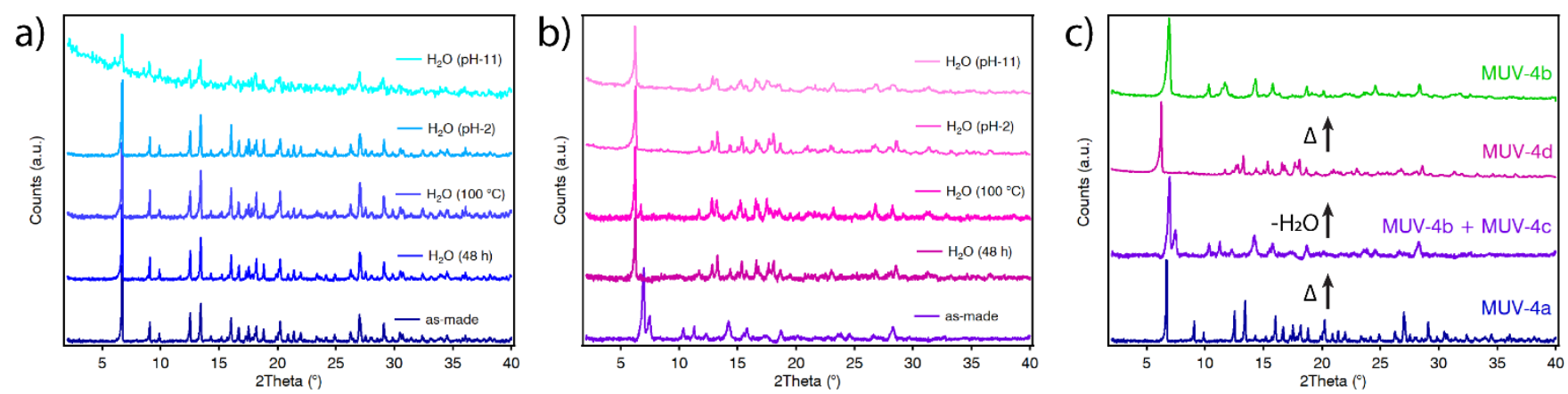

Figure 2 - Powder X-ray diffractograms of (a) MUV-4a and (b) MUV-4b (with presence of MUV-4c) after soaking in water under different conditions. (c) Different crystalline phases of MUV-4 obtained upon desolvation and subsequent rehydration.

The static magnetic susceptibility (Figure 3a) has been simulated with a relative error of $E=1.009 \cdot 10^{-4}$ and $E=$ $5.825 \cdot 10^{-5}$ for MUV-4a and MUV-4b, respectively, using the Radial Effective Charge (REC) model $^{30}$ in the SIMPRE computational package (see ESI for details). ${ }^{31}$ According to our calculations, the predicted ground state is in both cases mainly composed by $76 \%$ of $\mid \pm 15 / 2>$, which is in principle compatible with SMM behaviour (Table S4). However, a remarkable contribution $(7 \%)$ of $\mid \pm 1 / 2>$ and a lower first excited Kramers doublet (about $12 \mathrm{~cm}^{-1}$ ) are present in MUV-4a, which favours fast relaxation of the magnetization in this compound. On the other hand, MUV-4b presents a more favourable scenario to exhibit SMM behaviour as it does not present a significant contribution of $\mid \pm 1 / 2>$ and, moreover, the first excited energy level is located at $30 \mathrm{~cm}^{-1}$.

The results are contrary to the expected taking into account exclusively the geometrical features of the coordination
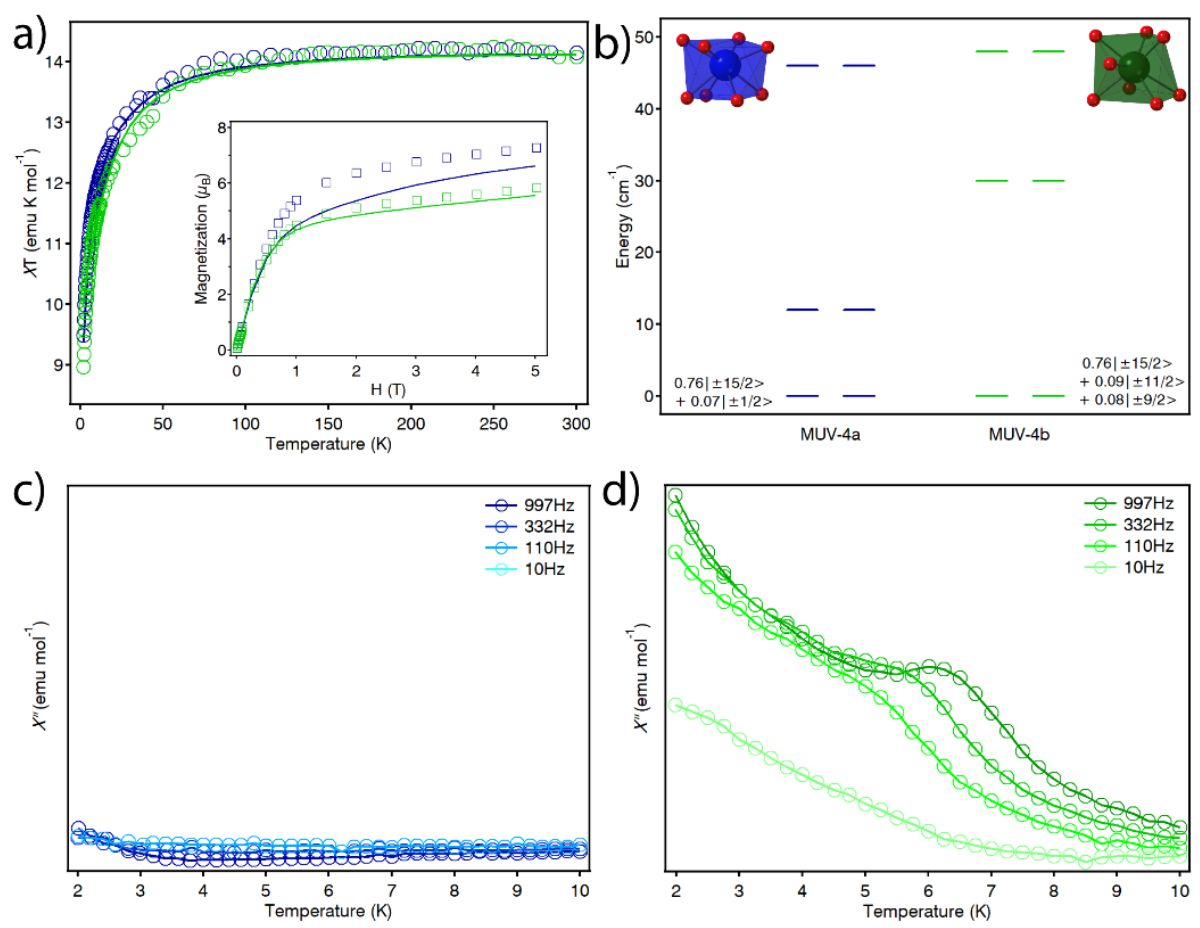

Figure 3 -(a) Experimental (symbols), fitted (solid line) temperature-dependence of the magnetic susceptibility from 2 to $300 \mathrm{~K}$. MUV-4a is shown in blue and MUV-4b is shown in green. The insets show experimental (symbols) and predicted (solid line) magnetization versus magnetic field at $2 \mathrm{~K}$. (b) Energy levels scheme with the main $M_{J}$ contributions to the ground state for MUV-4a and MUV-4b. (c,d) Out-of-phase dynamic magnetic susceptibility under an external magnetic field of $1000 \mathrm{G}$ for of MUV-4a (c) and MUV-4b (d). environment, as the octacoordinated axially elongated square antiprism geometry around the Dy(III) ion found in MUV-4a is very likely to stabilize a ground state characterized by a large $M_{J}$ value that allows the presence of slow magnetic relaxation at low temperature. ${ }^{14}$ On the contrary, the more distorted geometry of the octacoordinated Dy(III) centres of MUV-4b might indicate a less favourable situation to obtain SMM behaviour. Therefore, the different relaxation dynamics between MUV-4a and MUV-4b seem to be caused by the change of the charge distribution around the metal centres upon the dehydration process. This contrasts with a previously reported MOF where changes in the SMM behaviour observed upon dehydration are exclusively related to the large change in geometry observed, from octacoordinated to heptacoordinated Dy(III) centres. ${ }^{26}$

In order to elucidate if the differences in the electronic spin states and ground state composition mainly arise from the geometrical distribution of ligands around the Dy ions or from their different charge distribution, we replaced the two neutral $\mathrm{H}_{2} \mathrm{O}$ molecules in MUV-4a by two anionic carboxylate oxygen atoms in the model, while keeping the identical atomic positions. ${ }^{32}$ Thus, this hypothetical structure resultant from the replacement of water molecules by carboxylate oxygens has the same charge density as MUV-4b (8 carboxylate oxygens) but a different arrangement of donor atoms (square antiprism vs triangular dodecahedron). This resulted in a determined ground state formed by $86 \% \mid \pm 15 / 2>$ and a first excited state located at $18 \mathrm{~cm}^{-1}$ (Table S5), which suggests that the appearance of slow relaxation of the magnetization when passing from MUV-4a to MUV-4b is related to the charge density enhancement around the Dy(III) centres instead of due to the changes in the coordination environment. 
In order to demonstrate the differences in the magnetic behaviour of both derivatives, we have carried out dynamic magnetic measurements ( $a c)$. In the case of MUV-4a, no out-ofphase signal, $\chi^{\prime \prime}$, can be observed, even after applying an external magnetic $d c$ field (Figure 4c, S17). On the contrary, a frequency dependent out-of-phase signal is observed for MUV4b (Figure S18), as anticipated by the theoretical calculations. However, no maximum is observed above $2 \mathrm{~K}$, likely due to the presence of a very fast relaxation of the magnetization through a quantum tunnelling mechanism, as also suggested in previous SIM-MOFs, ${ }^{12}$ which can be avoided by applying an external magnetic $d c$ field. Thus, in the presence of a $d c$ magnetic field of $1000 \mathrm{G}$ a frequency dependent maximum is observed for $\chi^{\prime \prime}$ (Figure 4d). This can be observed more clearly for MUV-4d whose structure is expected to be similar to that of MUV-4b (Figure SI19).

\section{Conclusions}

In summary, we have shown a novel Dy-based MOF with a flexible coordination environment. The substitution of neutral ligands $\left(\mathrm{H}_{2} \mathrm{O}\right)$ by charged ligands (carboxylates) over two of the positions of the coordination sphere, maintaining a very similar geometry, controls the slow relaxation dynamics allowing the appearance of slow magnetic relaxation.

\section{Experimental}

\section{Materials and reagents}

5-nitroisophthalic acid (98\%), Dy(III) acetate hexahydrate (99.9 $\%), \mathrm{Gd}(\mathrm{III})$ acetate hexahydrate, sodium hydroxide (98\%), acetic acid (99.7\%) were purchased from Sigma-Aldrich, and used as received. $\mathrm{N}, \mathrm{N}$-dimethylformamide ( $\geq 99.8 \%$ ) was purchased from Scharlab. Ultrapure water from Milli-Q equipment was used when required. All reagents and solvents were used without any previous purification unless specified.

\section{Physical and Chemical Characterization}

Carbon, nitrogen and hydrogen contents were determined by microanalytical procedures using a LECO CHNS. Thermogravimetric analyses were carried out with a Mettler Toledo TGA/SDTA 851 apparatus between 25 and $800{ }^{\circ} \mathrm{C}$ under ambient conditions $\left(10^{\circ} \mathrm{C} \cdot \mathrm{min}^{-1} \mathrm{scan}\right.$ rate and an air flow of 30 $\left.\mathrm{mL} \cdot \mathrm{min}^{-1}\right)$. NMR ${ }^{1} \mathrm{H}$ spectra were run on a Bruker DRX300 spectrometer. XRD powder patterns were collected in a PANalytical X'Pert PRO diffractometer using copper radiation ( $\mathrm{Cu} \mathrm{K \alpha}=1.5418 \AA$ ) $)$ with an $\mathrm{X}^{\prime}$ Celerator detector, operating at 40 $\mathrm{mA}$ and $45 \mathrm{kV}$. Profiles were collected in the $2^{\circ}<2 \theta<40^{\circ}$ range with a step size of $0.013^{\circ}$.

\section{Synthesis of $3,3^{\prime}, 5,5^{\prime}$-azobenzenetetracarboxylic acid ( $\left.\mathrm{H}_{4} \mathrm{abtc}\right)$}

$\mathrm{H}_{4}$ abtc was synthesized according to a reported method. ${ }^{27}$ In a typical procedure, 5-nitroisophtalic acid (19 g) and sodium hydroxide ( $50 \mathrm{~g}$ ) were suspended in $250 \mathrm{~mL}$ of Milli-Q water and reacted at $60{ }^{\circ} \mathrm{C}$ with continuous stirring for 1 hour. Next, glucose (100 g) was dissolved in $100 \mathrm{~mL}$ of warm water and the resulting solution was added dropwise to the yellow slurry that became dark brown due to reduction of the nitro groups. The mixture was left to cool down for 30 minutes followed by exposure to an air stream for 16 hours with continuous stirring at room temperature. Next, the crude was cooled in an ice bath prior to isolation of the solid by filtration with vacuum. Finally, the solid was dissolved in $250 \mathrm{~mL}$ of water and acidified with $\mathrm{HCl}$ $37 \%$ to produce an orange precipitate. This was isolated by filtration, thoroughly washed with water and dried in an oven (92\% yield). Elemental analysis for $\mathrm{C}_{16} \mathrm{H}_{10} \mathrm{~N}_{2} \mathrm{O}_{8}$ : Calc. $\mathrm{C}(53.64), \mathrm{H}$ (2.81), N (7.86); found: C (53.59), H (3.02), N (7.75). Spectroscopic data matched those quoted in the literature. ${ }^{33}$

\section{Synthesis of MUV-4a}

$0.3 \mathrm{mmol}$ of $\mathrm{Dy}(\mathrm{OAc})_{3} \cdot 6 \mathrm{H}_{2} \mathrm{O}(123.6 \mathrm{mg})$ and $0.225 \mathrm{mmol}$ of $\mathrm{H}_{4}$ abtc $\left(80.7 \mathrm{mg}\right.$ ) were suspended in $12 \mathrm{~mL}$ of $\mathrm{H}_{2} \mathrm{O}$ in a $25 \mathrm{~mL}$ Schott bottle. To this suspension, $63 \mathrm{mmol}$ of acetic acid (3.6 $\mathrm{mL}$ ) was added. The mixture was then sonicated for a few seconds, placed in an oven and heated at $160{ }^{\circ} \mathrm{C}$ for 12 hours $\left(\uparrow+2.0^{\circ} \mathrm{C} \cdot \mathrm{min}^{-1}, \downarrow-0.4{ }^{\circ} \mathrm{C} \cdot \mathrm{min}^{-1}\right)$. Orange single crystals of MUV-4a were collected by filtration, washed thoroughly with DMF and water, and dried under vacuum at room temperature overnight. Yield: $79.8 \%$ (based on $\mathrm{H}_{4}$ abtc). Elemental analysis for $\left[\mathrm{Dy}\left(\mathrm{H}_{2} \mathrm{O}\right)_{2}(\mathrm{Habtc})\right] \cdot 1.7 \mathrm{H}_{2} \mathrm{O}$ : Calc. C (32.88), $\mathrm{H}(2.48), \mathrm{N}$ (4.79); found C (33.35), H (2.95), N (5.02).

\section{Synthesis of MUV-4b}

Aprox. $100 \mathrm{mg}$ of MUV-4a were heated under vacuum at $150^{\circ} \mathrm{C}$ for $2 \mathrm{~h}$ yielding a mixture of MUV-4b and MUV-4c, whose structures were solved by single crystal X-ray diffraction. However, the poor quality of the data corresponding to MUV4c was not good enough for a fully anisotropic refinement and therefore has not been deposited in the CCDC. Immersion in water of this mixture comprised by MUV-4b and MUV-4c during 2 days yields the new phase MUV-4d, which was characterized as a single phase by XRPD. Further heating to $150{ }^{\circ} \mathrm{C}$ under vacuum for $2 \mathrm{~h}$ yields phase pure MUV-4b. Elemental analysis for [Dy(Habtc)]: Calc. C (37.19), H (1.17), N (5.64); found C (37.70), H (1.08), N (5.64).

\section{Synthesis of Gd-MUV-4a}

Gd-MUV-4a was synthesized by following the same procedure described above for the synthesis of MUV-4a but using $\mathrm{Gd}(\mathrm{OAc})_{3} \cdot 6 \mathrm{H}_{2} \mathrm{O}$.

\section{X-ray single crystal diffraction}

A single crystal of each compound (MUV-4a and MUV-4b) was mounted on a on a cryoloop using a viscous hydrocarbon oil to coat the crystal. X-ray data were collected at $120 \mathrm{~K}$ on a Supernova diffractometer equipped with a graphitemonochromated Enhance (Mo) X-ray Source $(\lambda=0.71073 \AA$ ). The program CrysAlisPro, Oxford Diffraction Ltd., was used for unit cell determinations and data reduction. Empirical absorption correction was performed using spherical harmonics, implemented in the SCALE3 ABSPACK scaling algorithm. The crystal structures were solved and refined 
against all $F^{2}$ values by using the SHELXTL and Olex 2 suite of programs. ${ }^{34,35}$ Non-hydrogen atoms were refined anisotropically and hydrogen atoms were placed in calculated positions that were refined using idealized geometries (riding model) and assigned fixed isotropic displacement parameters. A summary of the data collection and structures refinements is provided in Table 1. CCDC-1855292 and -1855294 contain the supplementary crystallographic data for this paper. This data can be obtained free of charge from The Cambridge Crystallographic Data Centre via www.ccdc.cam.ac.uk/data_request/cif.

Table 1. Crystal data and structure refinement for MUV-4a and MUV-4b.

\begin{tabular}{|c|c|c|}
\hline & MUV-4a & MUV-4b \\
\hline Identification code & CCDC 1855292 & $\begin{array}{l}\text { CCDC } \\
1855294\end{array}$ \\
\hline Empirical formula & $\begin{array}{l}\mathrm{C}_{16} \mathrm{H}_{14.2} \mathrm{DyN}_{2} \mathrm{O}_{11} \\
.7\end{array}$ & $\begin{array}{l}\mathrm{C}_{16} \mathrm{H}_{11} \mathrm{DyN}_{2} \mathrm{O} \\
10\end{array}$ \\
\hline Formula weight & 584.48 & 553.77 \\
\hline Temperature (K) & $120(2)$ & $120(2)$ \\
\hline Crystal system & triclinic & triclinic \\
\hline Space group, Z & $P-1,2$ & $P-1,2$ \\
\hline a $(\AA ̊)$ & $6.6676(3)$ & $7.1771(2)$ \\
\hline$b(\AA ̊)$ & $10.1303(5)$ & $9.0830(3)$ \\
\hline$c(\AA)$ & $13.8039(6)$ & $13.2629(4)$ \\
\hline$\alpha\left({ }^{\circ}\right)$ & $74.983(4)$ & $109.487(3)$ \\
\hline$\beta\left({ }^{\circ}\right)$ & $78.546(4)$ & $90.285(2)$ \\
\hline$\gamma\left({ }^{\circ}\right)$ & $83.143(4)$ & $92.121(3)$ \\
\hline Volume $\left(\AA^{3}\right)$ & $880.33(7)$ & $814.38(5)$ \\
\hline$\rho_{\text {calc }}\left(\mathrm{g} / \mathrm{cm}^{3}\right)$ & 2.205 & 2.257 \\
\hline$\mu\left(\mathrm{mm}^{-1}\right)$ & 4.319 & 4.654 \\
\hline$F(000)$ & 568.0 & 526.0 \\
\hline Crystal size $\left(\mathrm{mm}^{3}\right)$ & $\begin{array}{l}0.157 \times 0.122 \times \\
0.055\end{array}$ & $\begin{array}{l}0.044 \times 0.035 \\
\times 0.021\end{array}$ \\
\hline Wavelength $(\AA ̊)$ & 0.71073 & 0.71073 \\
\hline $2 \Theta$ range $\left({ }^{\circ}\right)$ & $5.772-49.422$ & $\begin{array}{l}5.682- \\
49.426\end{array}$ \\
\hline Index ranges & $\begin{array}{l}-7 \leq h \leq 7,-11 \leq \\
k \leq 11,-16 \leq 1 \leq \\
16\end{array}$ & $\begin{array}{l}-8 \leq h \leq 8,-10 \\
\leq k \leq 10,-15 \leq \\
l \leq 15\end{array}$ \\
\hline Reflections collected & 9519 & 11040 \\
\hline Independent reflections & $\begin{array}{l}2996\left[R_{\text {int }}=\right. \\
0.0529, R_{\text {sigma }}= \\
0.0546]\end{array}$ & $\begin{array}{l}2785\left[R_{\text {int }}=\right. \\
0.0773, R_{\text {sigma }} \\
=0.0699]\end{array}$ \\
\hline $\begin{array}{l}\text { Data/restraints/paramet } \\
\text { ers }\end{array}$ & $2996 / 17 / 304$ & $2785 / 3 / 265$ \\
\hline Goodness-of-fit on $F^{2}$ & 1.071 & 1.039 \\
\hline Final $R$ indexes $[I>=2 \sigma(I)]$ & $\begin{array}{l}R_{1}=0.0262 \\
w R_{2}=0.0557\end{array}$ & $\begin{array}{l}R_{1}=0.0390, \\
w R_{2}=0.0776\end{array}$ \\
\hline Final $R$ indexes [all data] & $\begin{array}{l}\mathrm{R}_{1}=0.0290 \\
w R_{2}=0.0573\end{array}$ & $\begin{array}{l}R_{1}=0.0484, \\
w R_{2}=0.0817\end{array}$ \\
\hline $\begin{array}{l}\text { Largest diff. peak/hole / e } \\
\AA^{-3}\end{array}$ & $0.71 /-0.84$ & $1.21 /-1.03$ \\
\hline
\end{tabular}

\section{Conflicts of interest}

There are no conflicts to declare.

\section{Acknowledgements}

Financial support from the European Comission (ERC-2016-CoG 724681-S-CAGE and ERC-2016 Stg 714122 Chem-fs-MOF), the Spanish MINECO (Structures of Excellence María de Maeztu MDM-2015-0538, projects CTQ2014-59209-P, CTQ2017-89528P). G.M.E. and C.M.G. thank the Spanish MINECO for a Ramón y Cajal Fellowship. J.J.B. thanks the EU for a Marie Curie Fellowship (H2020-MSCA-IF-2016-751047). J.C.G. thanks MINECO for a predoctoral FPI grant (CTQ2014-59209-P).

\section{Notes and references}

1 G. Maurin, C. Serre, A. Cooper and G. Férey, Chem. Soc. Rev., 2017, 46, 3104-3107.

H. C. J. Zhou and S. Kitagawa, Chem. Soc. Rev., 2014, 43, 5415-5418.

3 H. Furukawa, K. E. Cordova, M. O'Keeffe and O. M. Yaghi, Sci. AAAS, 2013, 341, 1230444. P. Dechambenoit and J. R. Long, Chem. Soc. Rev., 2011, 40, 3249.

E. Coronado and G. Mínguez Espallargas, Chem. Soc. Rev., 2013, 42, 1525-1539.

6 G. Mínguez Espallargas and E. Coronado, Chem. Soc. Rev., 2018, 47, 533-557.

7

L. Thomas, F. Lionti, R. Ballou, D. Gatteschi, R. Sessoli and B. Barbara, Nature, 1996, 383, 145-147. M. N. Leuenberger and D. Loss, Nature, 2001, 410, 789793.

A. D. Katsenis, E. K. Brechin and G. S. Papaefstathiou, in Encyclopedia of Inorganic and Bioinorganic Chemistry, John Wiley \& Sons, Ltd, Chichester, UK, 2014, pp. 1-14. I.-R. Jeon and R. Clérac, Dalt. Trans., 2012, 41, 9569.

X. Zhang, V. Vieru, X. Feng, J.-L. Liu, Z. Zhang, B. Na, W. Shi, B.-W. Wang, A. K. Powell, L. F. Chibotaru, S. Gao, P. Cheng and J. R. Long, Angew. Chemie Int. Ed., 2015, 54, 98619865.

J. J. Baldoví, E. Coronado, A. Gaita-Ariño, C. Gamer, M. Giménez-Marqués and G. Mínguez Espallargas, Chem. - $A$ Eur. J., 2014, 20, 10695-10702. N. Ishikawa, M. Sugita, T. Ishikawa, S. Y. Koshihara and Y. Kaizu, J. Am. Chem. Soc., 2003, 125, 8694-8695.

M. A. AlDamen, J. M. Clemente-Juan, E. Coronado, C. Martí-Gastaldo and A. Gaita-Ariño, J. Am. Chem. Soc., 2008, 130, 8874-8875.

S. T. Liddle and J. Van Slageren, Chem. Soc. Rev., 2015, 44, 6655-6669.

16 L. Escalera-Moreno, J. J. Baldoví, A. Gaita-Ariño and E. Coronado, Chem. Sci., 2018, 9, 3265-3275.
J. D. Rinehart and J. R. Long, Chem. Sci., 2011, 2, 2078.

J. J. Baldoví, S. Cardona-Serra, J. M. Clemente-Juan, E. Coronado, A. Gaita-Ariño and A. Palii, Inorg. Chem., 2012, 51, 12565-12574. 

2017, 56, 11445-11449.

21 K. Liu, X. Zhang, X. Meng, W. Shi, P. Cheng and A. K. Powell, Chem. Soc. Rev., 2016, 45, 2423-2439. Q. Chen, J. Li, Y. S. Meng, H. L. Sun, Y. Q. Zhang, J. L. Sun and S. Gao, Inorg. Chem., 2016, 55, 7980-7987. I. Oyarzabal, B. Fernández, J. Cepeda, S. Gómez-Ruiz, A. J. Calahorro, J. M. Seco and A. Rodríguez-Diéguez, CrystEngComm, 2016, 18, 3055-3063. K. Liu, H. Li, X. Zhang, W. Shi and P. Cheng, Inorg. Chem., 2015, 54, 10224-10231.

G. Huang, G. Fernandez-Garcia, I. Badiane, M. Camarra, S. Freslon, O. Guillou, C. Daiguebonne, F. Totti, O. Cador, T. Guizouarn, B. LeGuennic and K. Bernot, Chem. - A Eur. J., 2018, 24, 6983-6991.

26 Q. Zhou, F. Yang, B. Xin, G. Zeng, X. Zhou, K. Liu, D. Ma, G. Li, Z. Shi and S. Feng, Chem. Commun., 2013, 49, 82448246. P. S. Z. Sardar Ameerunisha, J. Chem. Soc. Perkin Trans. 2, 1995, 1679.

28 J.-P. Zhang, P.-Q. Liao, H.-L. Zhou, R.-B. Lin and X.-M. Chen, Chem. Soc. Rev., 2014, 43, 5789-5814.

29 A. Schneemann, V. Bon, I. Schwedler, I. Senkovska, S. Kaskel and R. A. Fischer, Chem. Soc. Rev., 2014, 43, 60626096.

30 J. J. Baldoví, J. J. Borrás-Almenar, J. M. Clemente-Juan, E. Coronado and A. Gaita-Ariño, Dalton Trans., 2012, 41, 13705-13710 J. J. Baldoví, S. Cardona-Serra, J. M. Clemente-Juan, E. Coronado, A. Gaita-Ariño and A. Palii, J. Comput. Chem., 2013, 34, 1961-1967. K. S. Lim, J. J. Baldoví, W. R. Lee, J. H. Song, S. W. Yoon, B. J. Suh, E. Coronado, A. Gaita-Ariño and C. S. Hong, Inorg. Chem., 2016, 55, 5398-5404. C. Qin, Y. Feng, H. An, J. Han, C. Cao and W. Feng, ACS Appl. Mater. Interfaces, 2017, 9, 4066-4073. G. M. Sheldrick, Acta Crysta llogr. Sect. A 2008, 64, 112122.

O. V. Dolomanov, L. J. Bourhis, R. J. Gildea, J. A. K. Howard, H. Puschmann, J. Appl. Crystallogr. 2009, 42, 339-341. 